\title{
Rolling Acrobatic Apparatus
}

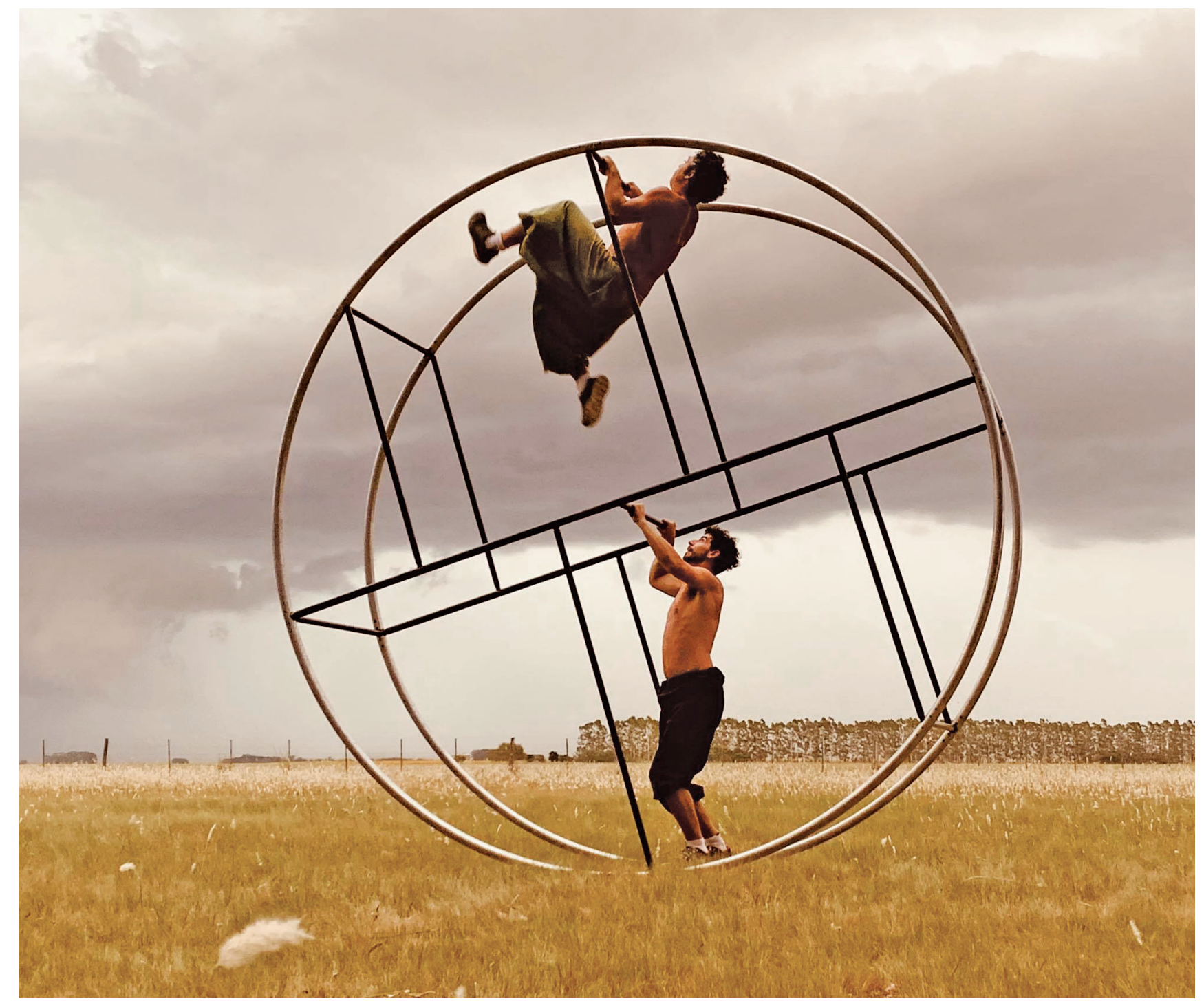

\section{Henry Segerman}

\section{Introduction}

A popular class of acrobatic discipline revolves around a performer or performers controlling the movement of a large metal frame by shifting their body weight within it, causing it to roll across the stage. One of the most common variants is the German wheel. The German wheel has

Henry Segerman is an associate professor of mathematics at Oklahoma State University. His email address is segerman@math. okstate. edu.

For permission to reprint this article, please contact:

reprint-permission@ams.org.

DOI: https://doi.org/10.1090/noti2312 two parallel rings joined by a number of rungs and handles that the performers hold. The image at the beginning of this article shows a large German wheel, intended for multiple performers. A less well-known kind of apparatus is the Zigrolling, invented by Toni Vighetto, shown in Figure 1. See https://bit.7y/3wgmvEP for a video of the Zigrolling in motion. This acts in essentially the same way as the sphericon [3], which is a solid formed from two right-angled cones as described in Figure 2. Figure 3 shows how the Zigrolling and sphericon roll on the plane: they "zigzag," pivoting on an endpoint of one semicircle while rolling along the other, then vice versa. 


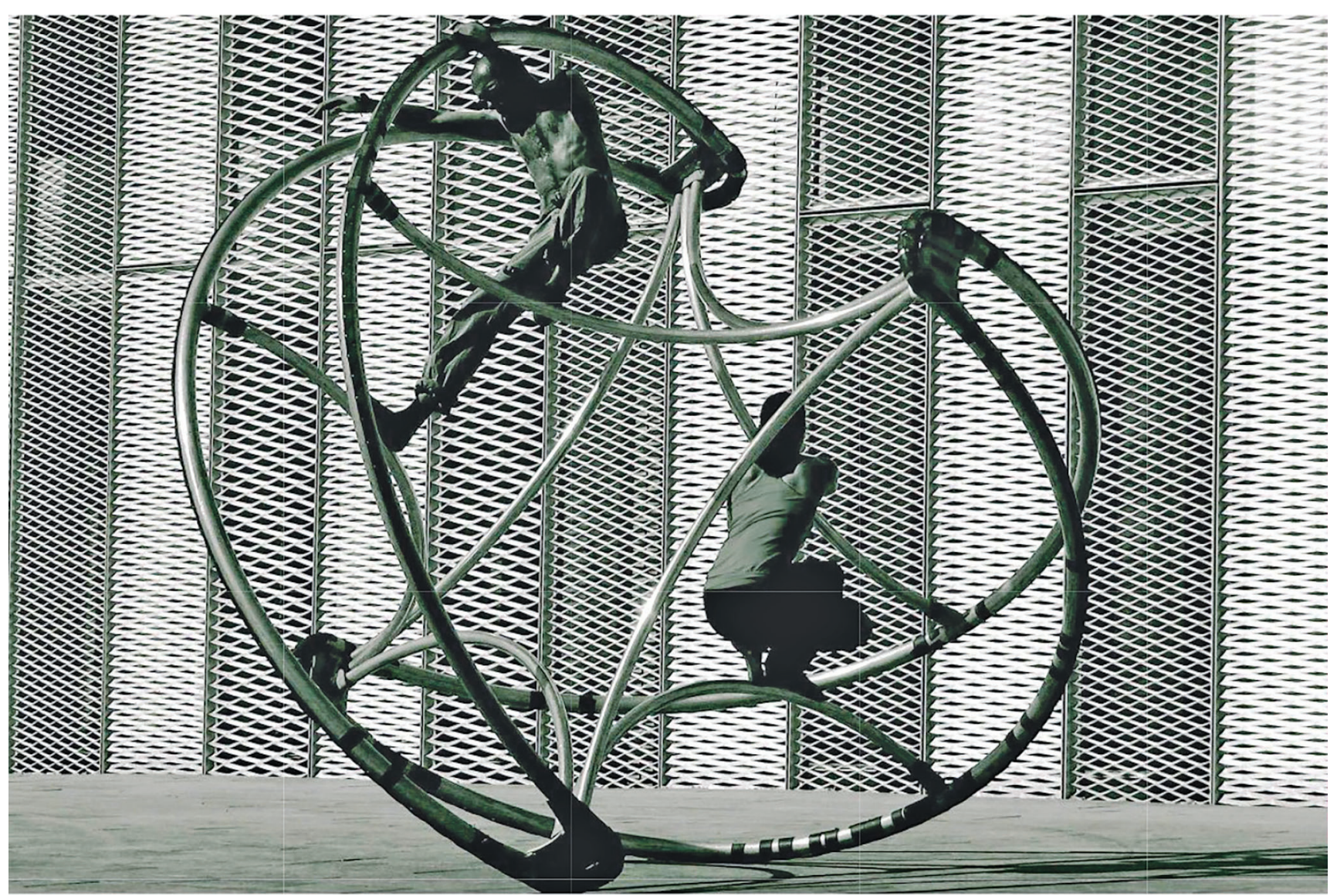

Figure 1. Toni Vighetto (top) and Marie-Martine Robles on a Zigrolling apparatus.

In the Zigrolling apparatus, the semicircular arcs of the sphericon are replaced by loops closely approximating them. As a piece of notation, we refer to the parts of an apparatus that contact the stage as the apparatus moves as the rails. The remainder of the structure connects the rails together and provides geometry for the performers to step or hold on to.

I first met Marco Paoletti, a world-renowned juggler and circus performer, at the Melbourne Juggling Convention in 2012. Marco is at the top of the German wheel in the image at the beginning of this article (Matias D'Angelo is below). He told me about the Zigrolling apparatus, and the following problem: although it does zigzag as it travels across the stage, after one full cycle it is facing in the same direction as when it started. On the large scale then, it travels in a straight line, just like the German wheel. In performance, the acrobat runs out of room on the stage and must stop and go backwards, breaking the flow of forward motion. Marco asked me if the apparatus could be redesigned to fix this: for example, perhaps if the two semicircles of the $\mathrm{Zi}$ grolling apparatus were different sizes, then the apparatus would go around in a circle while zigzagging? The purpose of this paper is to answer this question, and suggest some alternative apparatus designs.

\section{Construction of Rolling Apparatus}

We make the following assumption about our rolling apparatus.

Assumption 2.1. The center of mass of the apparatus does not change height as it rolls.

This is not necessarily the case for every usable rolling metal apparatus, but it is (at least approximately) the case for the German wheel and the Zigrolling apparatus. If this assumption is not satisfied, then there will be points in the motion of the apparatus for which the height of the center of mass is at a local minimum. Movement away from such a point requires work on the part of the performer to "climb out of the valley." Such a climb requires sufficient friction between the apparatus and the stage to avoid slipping. The performer may not easily be able to hold the apparatus at a particularly steep point, and may have no choice but to let it roll downhill. All of these issues restrict the ways in which the performer can use the apparatus.

If we follow Assumption 2.1, then the performer has control of the motion for all configurations of the apparatus. They need only make small changes to their position to shift the overall center of mass of the apparatus and the performer, and so control the apparatus. 


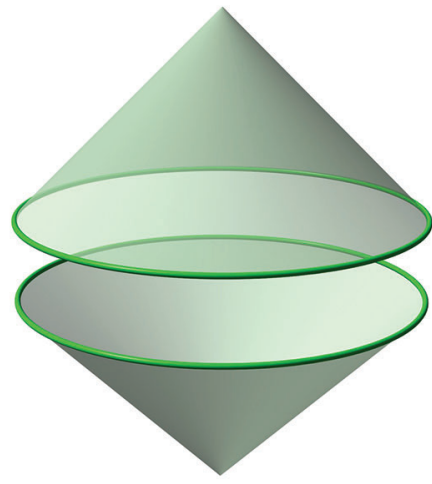

(A) Start with two right cones.

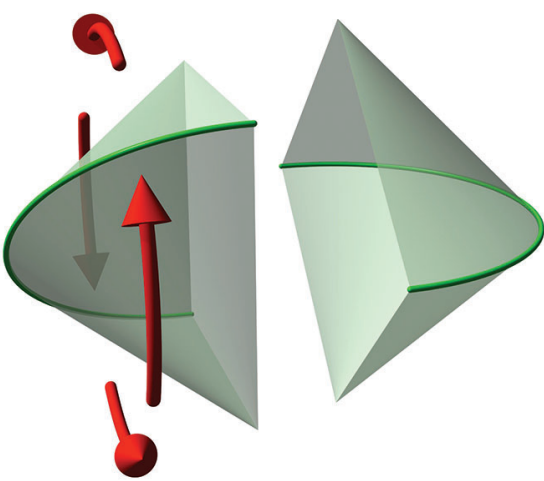

(D) Rotate one half...

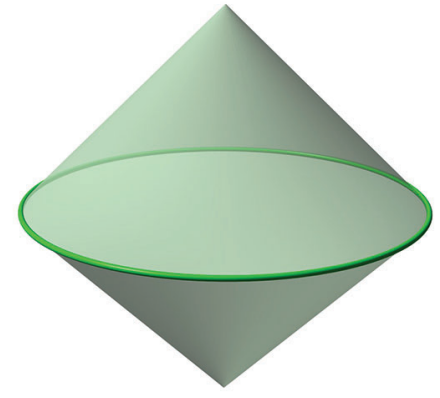

(B) Glue together along their bases.

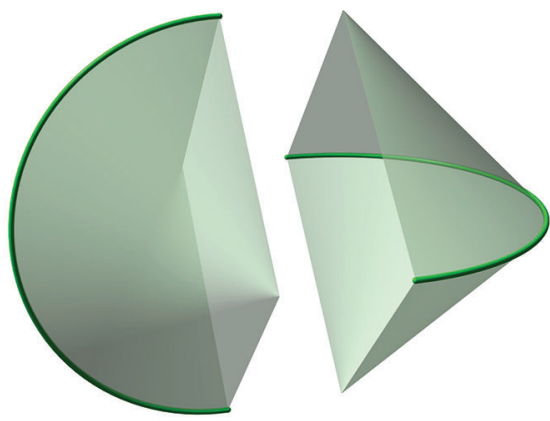

(E) ...by a quarter turn.

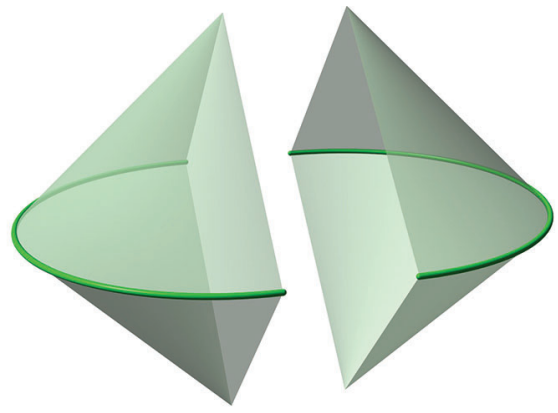

(C) Cut with a vertical plane.

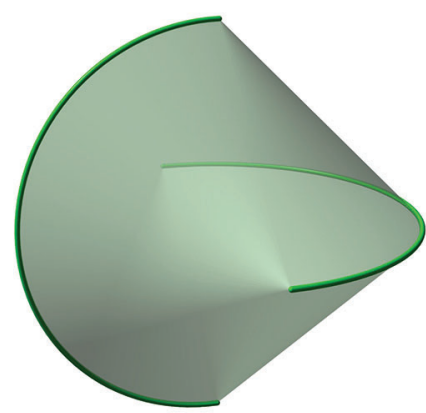

(F) Glue together.

Figure 2. Construction of the sphericon.

2.1. The rolling sphere. With Assumption 2.1 in hand, we attach an imaginary sphere to the apparatus, of radius the height of its center of mass above the stage. We call this the rolling sphere. At each point in the movement of an apparatus, the rolling sphere touches the stage in a single point. Tracing these points out as the apparatus rolls, we produce two curves: $\sigma$ on the rolling sphere and $\rho$ on the plane of the stage. See Figure 4 for an example where $\sigma$ is the "tennis ball" curve, made from four congruent semicircles.

In general, we will assume that $\sigma$ is a piecewise smooth simple closed curve. From now on, we assume that the rolling sphere has radius one, and that $\mathbf{s}(t)$ is a unit speed parametrization of $\sigma$. Let $\mathbf{r}(t)$ be the corresponding unit speed parametrization of $\rho$ on the plane. When we are working in the frame of reference of the apparatus rather than the stage, we arrange matters so that the rolling sphere is centered at the origin.

2.2. Ruled surfaces. We construct a set of rails for an apparatus that rolls along a given curve $\sigma$ on the rolling sphere, as follows. First, we construct a ruled surface,
$S(t, u)$, tangent to the rolling sphere, made up of lines that are perpendicular to both $\mathbf{s}(t)$ (a vector from the center of the sphere to its surface) and to $\mathbf{s}^{\prime}(t)$. In other words, for fixed $t_{0}, S\left(t_{0}, u\right)$ is a line given by

$$
S\left(t_{0}, u\right)=\mathbf{s}\left(t_{0}\right)+u \mathbf{b}\left(t_{0}\right)
$$

where $\mathbf{b}\left(t_{0}\right)=\mathbf{s}\left(t_{0}\right) \times \mathbf{s}^{\prime}\left(t_{0}\right)$ is a unit vector perpendicular to both $\mathbf{s}\left(t_{0}\right)$ and $\mathbf{s}^{\prime}\left(t_{0}\right)$. For differential geometers: this construction naturally takes place in the Darboux frame for $\sigma$ embedded in the rolling sphere. A straightforward calculation using the fact that $\mathbf{s}(t) \times \mathbf{s}^{\prime \prime}(t)=0$ shows that the normal to the surface $S(t, u)$ does not depend on $u$. It follows from this that the gaussian curvature of $S(t, u)$ is zero, and so $S(t, u)$ is developable [4, p. 91].

With a sufficiently nice curve $\sigma$, truncating these lines $S\left(t_{0}, u\right)$ where they intersect lines coming from other points on $\sigma$ produces a closed surface, $S$. In the example shown in Figure 4, the closed surface generated by the "tennis ball" curve on the rolling sphere is the sphericon. See Figure 5A. 


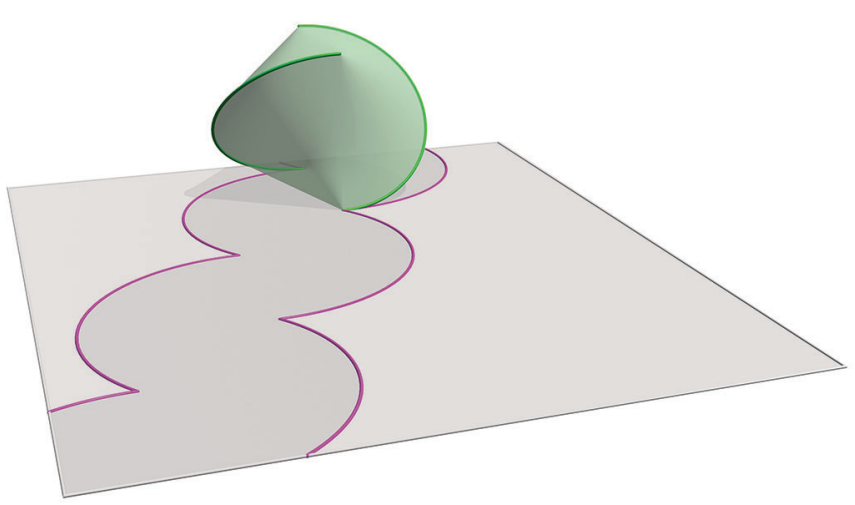

Figure 3. The sphericon rolls along a zigzag path.

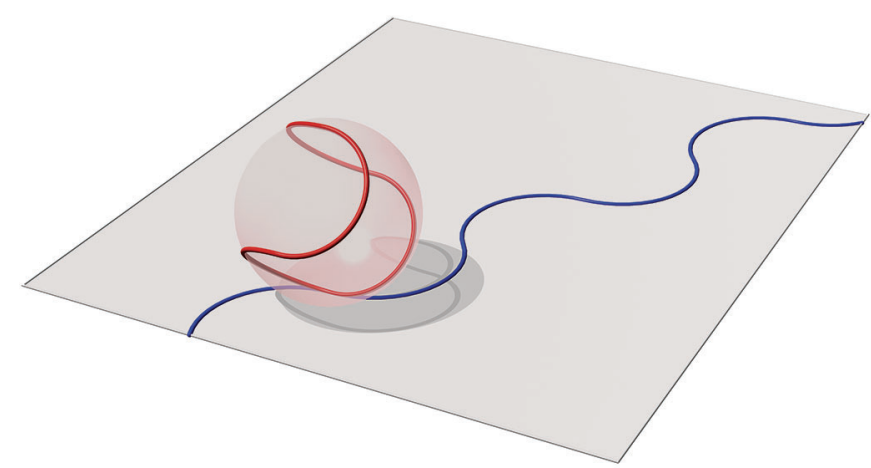

Figure 4. The curves $\sigma$ (on the rolling sphere) and $\rho$ (on the plane).

A closed surface works well as the boundary of a solid object, as for the sphericon. For an acrobatic apparatus we replace the ruled surface $S$ with a collection $K$ of onedimensional rails that rolls along $\sigma$ in the same way. We may take for this collection the set of points of intersection of the ruled surface $S(t, u)$ with itself. In Figure 5A, these arcs of self-intersection are the two semicircles of the sphericon. The two loops of the Zigrolling apparatus are another collection of rails that rolls along $\sigma$. In general, the requirement for $K$ is that for each line $S\left(t_{0}, u\right)$, the set $K \cap S\left(t_{0}, u\right)$ must contain a point on either side of $\sigma \cap S\left(t_{0}, u\right)$. This ensures that the apparatus will not tip over in the plane containing the center of the rolling sphere and the line $S\left(t_{0}, u\right)$.

In our example, we must add some extra structure to connect the two semicircular rails together-in Figure 5B we have included four rulings of the ruled surface forming a square. For a full-size physical apparatus, these rulings would not be suitable. In Figure 1 they are replaced by connecting arcs that curve in towards the center of the apparatus. These are easier for the performer to hold on to, and remove the danger of the performer gripping a bar which contacts the floor, resulting in fingers getting run over!

Figures $5 \mathrm{C}$ to $5 \mathrm{E}$ show three positions of the apparatus as it rolls along an arc.

\section{How Big Does the Stage Need to Be?}

In order to answer Marco's question, we need to understand the movement on the plane of the stage corresponding to rolling around the apparatus. In particular, we want to know whether the curve on the stage, $\rho$, is bounded. Since the curve $\sigma$ is closed, this comes down to understanding the difference in the position and facing of the apparatus after rolling around $\sigma$ once. This isometry of the plane is called the holonomy of $\sigma$, denoted $I(\sigma)$. We then have the following.

Lemma 3.1. The path $\rho$ is unbounded if and only if the holonomy $I(\sigma)$ is a translation by a non-zero distance.

Proof. First note that if $I(\sigma)$ is a translation, then repeatedly rolling the apparatus around $\sigma$ repeats the translation, and the apparatus will eventually reach the edge of any stage. If on the other hand $I(\sigma)$ is a rotation, then although the path $\rho$ may never return to precisely the same position and facing as at the start, it cannot move further from the center of that rotation than it did on the first trip around $\sigma$. Thus a stage of some finite diameter will contain $\rho$.

So, to answer Marco's question, we need to understand the relationship between the curve $\sigma$ and the angle that the apparatus turns after rolling around $\sigma$. In fact, we have the following result.

Theorem 3.2. The turning angle of the apparatus after rolling once around the simple closed curve $\sigma$ is the difference between $2 \pi$ and the area of either component of the sphere minus $\sigma$.

Note that since the total area of the sphere is $4 \pi$, the two differences are the same up to sign. The outline of the proof is to use Gauss-Bonnet to show that the area difference is equal to the total geodesic curvature of $\sigma$, which is the same as the total geodesic curvature of a period of $\rho$, which is zero if and only if the holonomy is a translation.

Proof. As the rolling sphere rolls on the plane, we roll the ruled surface along with it. This produces a one-parameter family of lines $R(t, u)$ on the plane, made from the positions of lines $S(t, u)$ as they become tangent to the plane.

For example, if a segment of $\sigma$ is an arc of a circle, then the corresponding subset of $S(t, u)$ is part of a cone. The corresponding subset of the one-parameter family of lines $R(t, u)$ is part of a disk. See Figure 6 . In this case it is clear that $S(t, u)$ is isometric to $R(t, u)$, and in fact this is true in general: developable surfaces are isometric to the plane [4, Section 5-3].

We recall the definition of geodesic curvature. Let $\mathbf{c}(t)$ be a smooth unit speed path on a surface $F$ in $\mathbb{R}^{3}$. Let $\mathbf{n}(t)$ be the normal to $F$ at $\mathbf{c}(t)$. Then $\mathbf{c}^{\prime}(t)$ and $\mathbf{n}(t)$ are perpendicular, and so $\left\{\mathbf{c}^{\prime}(t), \mathbf{n}(t), \mathbf{n}(t) \times \mathbf{c}^{\prime}(t)\right\}$ form an orthonormal basis for $\mathbb{R}^{3}$. Thus the second derivative $\mathbf{c}^{\prime \prime}(t)$ can be written as a linear combination of $\mathbf{c}^{\prime}(t), \mathbf{n}(t)$, and 


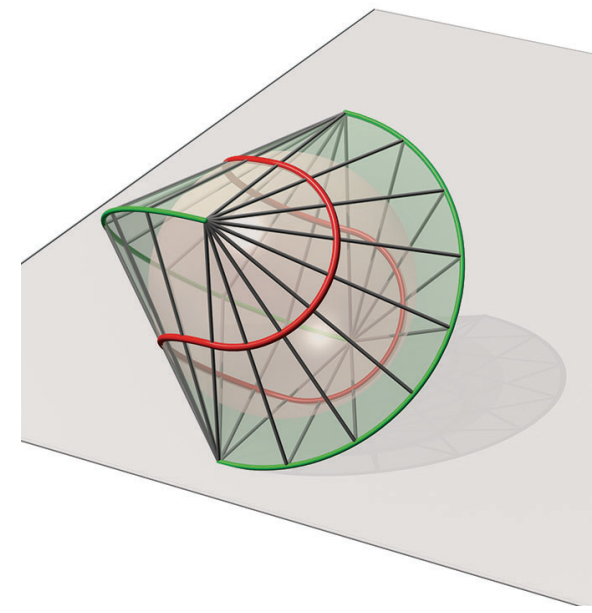

(A) A sphericon generated from ruled surfaces.

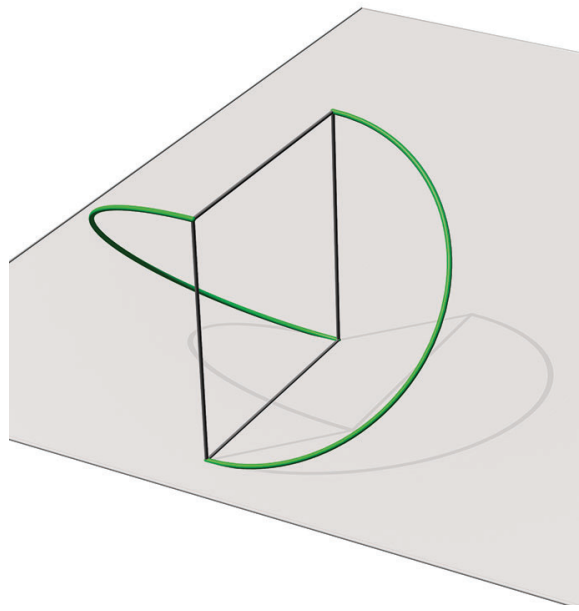

(B) Taking the curves of intersection between the ruled surfaces approximates the Zigrolling apparatus.

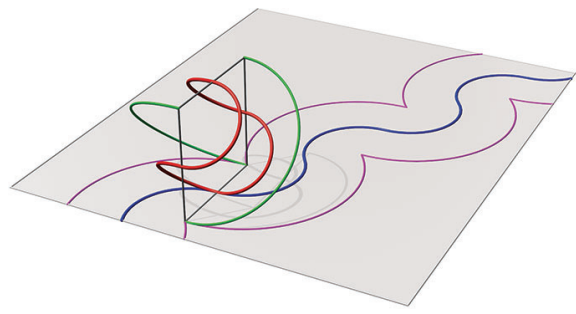

(C)

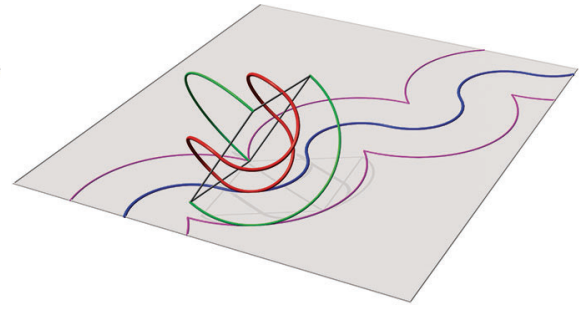

(D)

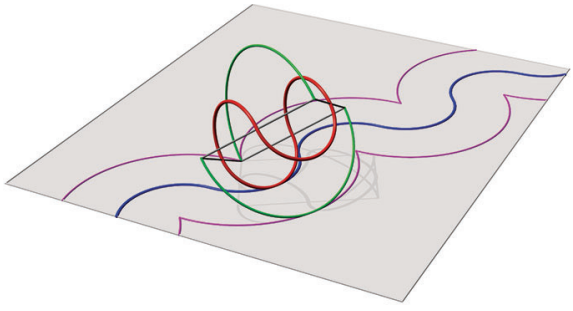

(E)

Figure 5. Throughout the paper, the curve $\sigma$ is drawn in red and $\rho$ is drawn in blue. The rails of the apparatus are drawn in green. Rulings used to connect the rails together are drawn in grey. When drawn, the paths that the rails roll along on the stage are drawn in magenta.

$\mathbf{n}(t) \times \mathbf{c}^{\prime}(t)$. However, $\mathbf{c}^{\prime \prime}(t)$ is perpendicular to $\mathbf{c}^{\prime}(t)$ since $\mathbf{c}(t)$ is unit speed. So we can write:

$$
\mathbf{c}^{\prime \prime}(t)=\kappa_{n}(t) \mathbf{n}(t)+\kappa_{g}(t)\left(\mathbf{n}(t) \times \mathbf{c}^{\prime}(t)\right) .
$$

Here, $\kappa_{n}(t)$ is the normal curvature, and $\kappa_{g}(t)$ is the geodesic curvature of $\mathbf{c}$ at time $t$. It is a standard fact that geodesic curvature is intrinsic. In other words, it is invariant under isometries of the surface. In particular, the geodesic curvature of $\mathbf{s}(t)$ on $S(t, u)$ is equal to the geodesic curvature of $\mathbf{r}(t)$ on $R(t, u)$.

Recall that $\sigma$ is simple. Thus it separates the rolling sphere into two disks, say $P$ to the left of $\sigma$, and $Q$ to the right. Next we apply the Gauss-Bonnet theorem to $P$ :

$$
\int_{P} K d A+\int_{\partial P} \kappa_{g} d s=2 \pi \chi(P)
$$

Here the second integral is interpreted as the sum of the corresponding integrals along smooth pieces of $\partial P$, plus the sum of the angles by which the smooth pieces turn at corners.

The Gaussian curvature $K$ is one, the boundary of the disk $\partial P$ is $\sigma$, and the Euler characteristic $\chi(P)=1$, so after rearranging we get

$$
2 \pi-\operatorname{Area}(P)=\int \kappa_{g} d s .
$$

Now, since the geodesic curvature of $\sigma$ on $S(t, u)$ is equal to the geodesic curvature of $\rho$ on $R(t, u)$, we can view $\kappa_{g}$ as the geodesic curvature on $R(t, u)$. Since $\rho$ is a curve in the plane, it has zero normal curvature, so $\kappa_{g}$ is just the signed curvature of the planar curve $\rho$. Again, the integral is interpreted as being the sum of the integrals along smooth pieces, plus the sum of the angles by which the smooth pieces turn at corners. Note that the sum of the angles turned at any corners is the same on the plane as it is on the sphere. Thus the right-hand side of this equation calculates the total change of direction of the curve $\rho$ as we go once around $\sigma$. This gives us Theorem 3.2.

\section{Marco's Question}

Applying Theorem 3.2, the turning angle is zero when $\operatorname{Area}(P)=2 \pi$. That is, precisely half of the total area of the (unit) rolling sphere. A turning angle of some other integer multiple of $2 \pi$ is not possible, since then we would have the area of either $P$ or $Q$ being less than or equal to 


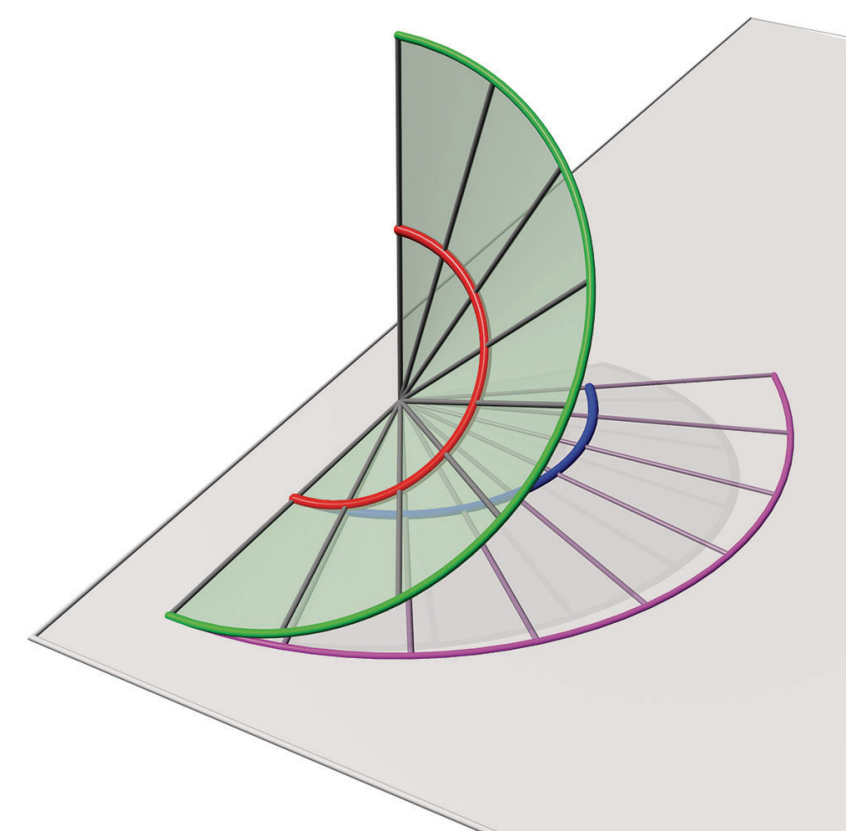

Figure 6. Part of $S(t, u)$ and the corresponding part of $R(t, u)$.

zero. This is impossible without either self-intersection, or the curve $\sigma$ being trivial.

We therefore have the following characterization of whether or not an apparatus rolling on a simple closed curve $\sigma$ on the rolling sphere can roll forever on a bounded stage.

Corollary 4.1. A rolling apparatus requires an unbounded stage if and only if the curve $\sigma$ divides the rolling sphere into two regions of equal area.

This is the case for the Zigrolling apparatus with its "tennis ball" curve, as shown in Figure 5. If we instead want a specified turning angle, then we must choose a curve with the corresponding area cut out by our curve $\sigma$.

There is a one-parameter family of deformed tennis ball curves, each made from four semicircles, their endpoints meeting a great circle at the corners of a rectangle. See Figure 7A. This time we have two different radii of semicircles, whose squares sum to one. If our semicircles have radii $a$ and $b$ with $a^{2}+b^{2}=1$, then we can calculate the areas of the two components of the sphere as follows.

The total area of the sphere is $4 \pi$, which is made up of two spherical "caps" with boundary a circle of radius $a$, two caps with boundary a circle of radius $b$, and the remainder, consisting of two cusped rectangles. The area of the radius $a$ cap is

$$
\begin{aligned}
& \int_{0}^{\arcsin a} \int_{0}^{2 \pi} \sin \phi d \theta d \phi \\
& =2 \pi(1-\cos \arcsin a) \\
& =2 \pi\left(1-\sqrt{1-a^{2}}\right)=2 \pi(1-b) .
\end{aligned}
$$

So the total area of the two cusped rectangles is

$$
4 \pi-2(2 \pi(1-b))-2(2 \pi(1-a))=4 \pi(a+b-1) \text {. }
$$

Thus, the area of the component on the side with the radius $a$ caps is

$$
2(2 \pi(1-b))+\frac{1}{2}(4 \pi(a+b-1))=2 \pi(1+a-b),
$$

so the turning angle of the apparatus is $2 \pi(a-b)$. Given a desired turning angle, we can then solve for $a$ and $b$. For example, if we want the turning angle to be $2 \pi / 3$, then we should take $a=(\sqrt{17}-1) / 6, b=(\sqrt{17}+1) / 6$. A design using these parameters is shown in Figure $7 \mathrm{~B}$.

This apparatus stays on the stage as desired, but it may be difficult to satisfy our assumption that the center of mass is at the origin of the rolling sphere. Certainly with metal tube of constant density, the design shown in Figure 7B will not have its center of mass at the center of the rolling sphere. In principle this could be fixed with some kind of added weight. Alternatively, perhaps some other closed curve that unequally divides the rolling sphere could be designed, in such a way that the center of mass of the rails is at the origin. But even then careful thought would have to be put into the rest of the structure to maintain this property, and it is hard to imagine how to do this elegantly.

4.1. Symmetry and center of mass. In general, it will be a difficult task to produce a design whose center of mass is at the origin. Mathematically, one might proceed with an integral of some form, assuming constant density. However, a real-life construction may have more mass at junctions of the structure where more strength is required, or have added material to improve the grip of the apparatus on the stage, or various harnesses for performers to strap into, and so on. However, there is a convenient criterion based on symmetry that will ensure that the final physical apparatus has its center of mass at the origin.

Theorem 4.2. If the symmetry group of an apparatus pointwise fixes only the origin $O$ of the rolling sphere, then the center of mass of the apparatus is $O$.

Proof of Theorem 4.2. By definition, an object is fixed by any element of its symmetry group. Therefore the object's center of mass is also fixed. Under the hypotheses of the theorem, the center of mass must be at $O$.

Perhaps the most convenient notation system for the symmetries of (bounded) three-dimensional objects is the Conway-Thurston orbifold notation [1]. See the sidebar below for a brief introduction. The symmetry types that fix more than just the origin are $1, n n$, and ${ }^{*} n n$ for $n \geq 1$. As an example, the original Zigrolling apparatus (with the standard tennis ball curve) has symmetry type $2 * 2$ (see Figure $8 \mathrm{C}$ ), so its center of mass is at the origin. Our less symmetric Zigrolling apparatus (with a modified tennis ball 


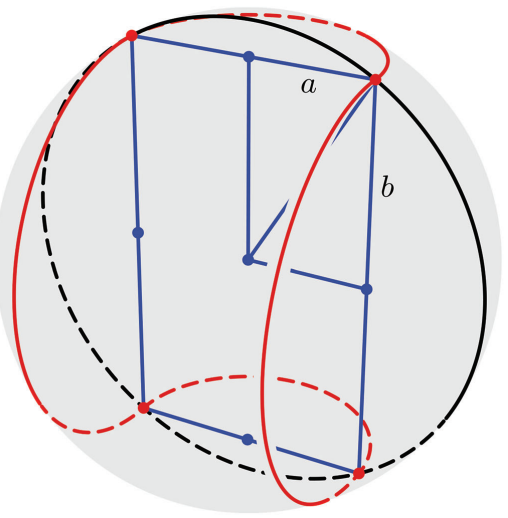

(A) A less symmetric tennis ball curve.

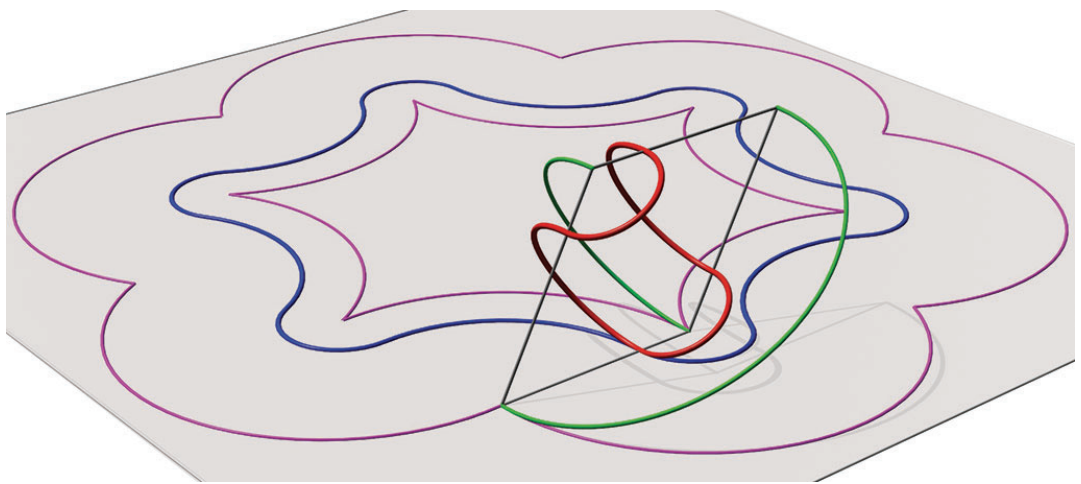

(B) A less symmetric Zigrolling apparatus that comes back to the same position after three cycles.

Figure 7. Construction of a less symmetric Zigrolling apparatus.

curve) has symmetry type *22 (see Figure 8 F), and so it will not, unless very careful thought is put into the construction.

Sidebar: Conway-Thurston orbifold notation. This notation efficiently describes finite subgroups of $O(3)$, the isometries of $\mathbb{R}^{3}$ that fix the origin, by describing orbifold quotients of the sphere, $S^{2}$. This naturally extends to symmetries in $\mathbb{E}^{2}$ and $\mathbb{\boxplus}^{2}$.

The notation consists of a sequence of digits, possibly including a ${ }^{*}$ or $\times$ symbol. In the absence of such a symbol, a digit $n$ corresponds to a point on the sphere with $n$-fold rotational symmetry. In Figure $8 \mathrm{~A}$, there are two different points with 8 -fold rotational symmetry, so the notation is 88 . The presence of $\mathrm{a}^{*}$ indicates that there are planes of mirror symmetry. A digit $n$ after a ${ }^{*}$ corresponds to a point on the sphere with $n$ planes of mirror symmetry passing through it. In Figure 8B, there are two different points with 4 mirror planes, so the notation is $* 44$. A digit before a $*$ corresponds to a point with rotational symmetry as before: Figure $8 \mathrm{C}$ has a point with 2 -fold rotational symmetry, and a point with 2 mirror planes, so the notation is $2^{*} 2$. Note that we only count points up to symmetry: the four rotationally symmetric points here are all the same as each other, as are the two points with 4 mirror planes. In Figure 8D we have (up to symmetry) a point with 8 -fold rotational symmetry, and a single mirror plane. This is denoted $8^{*}$, with no digit after the *. Finally, the presence of an orientation-reversing isometry that is not a reflection is denoted with $\mathrm{a} \times$. So for example, Figure $8 \mathrm{E}$ has (up to symmetry) a point with 8 -fold rotational symmetry, and a symmetry that is a composition of a rotation and a reflection.
The complete list of spherical symmetry types is $332, * 332,3^{*} 2,432,{ }^{*} 432,532,{ }^{*} 532, n n, n \times, n^{*},{ }^{*} n n$, $22 n, 2^{*} n$, and ${ }^{*} 22 n$ for $n \geq 1$ an integer. That these are all of the possibilities can be efficiently proved using orbifold Euler characteristic [1].

One might hope that some other design could unequally divide the sphere while having a symmetry type that ensures that the center of mass is at the origin. Unfortunately this is impossible, as the next result shows.

Theorem 4.3. Suppose that $\sigma$ is a piecewise-smooth simple closed curve on the sphere. If $\sigma$ divides the sphere into two nonisometric disks, then the symmetry group $G$ of $\sigma$ pointwise fixes more than only the origin of the sphere.

Proof. Let $H<G$ be the orientation-preserving subgroup. First suppose that $H$ is trivial. If $G=H$, then it fixes everything and we are done. Otherwise, $G$ is generated by an order two element: either a reflection or the antipodal map. In the former case (symmetry type ${ }^{*}$ ), $G$ fixes the plane of the reflection and we are done. In the latter (symmetry type $\times$ ), the antipodal map swaps the complementary components of $\sigma$ so they are isomorphic, contradicting the hypothesis.

Claim. Let $g \in H-\{1\}$. Then $g$ is a rotation. Also, the loop $\sigma$ misses the fixed points of $g$.

Proof of Claim. By Euler's theorem, all non-trivial orientation-preserving isometries of the sphere are rotations. For a contradiction, suppose that $p \in \sigma$ is a fixed point of $g$. Since $\sigma$ is simple, it splits a small neighbourhood of $p$ into two components. However, $g$ cannot interchange these two components since the two complementary disks of $\sigma$ are not isometric.

Next suppose that $H$ is finite and non-trivial. We now prove that $H$ is cyclic. By the claim and since $H$ is finite, 


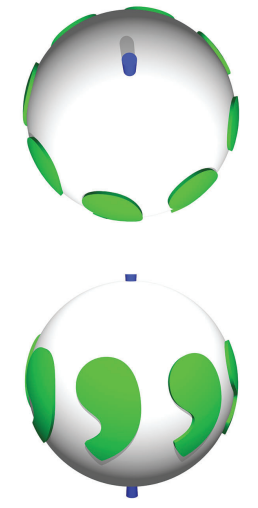

(A) 88

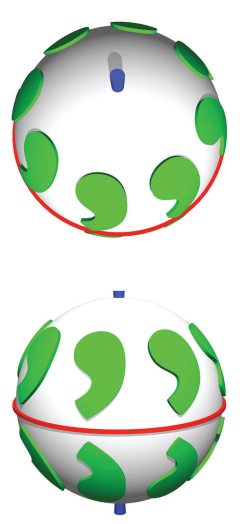

(D) $8^{*}$
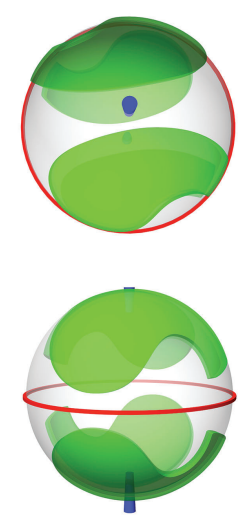

(G) $2 *$
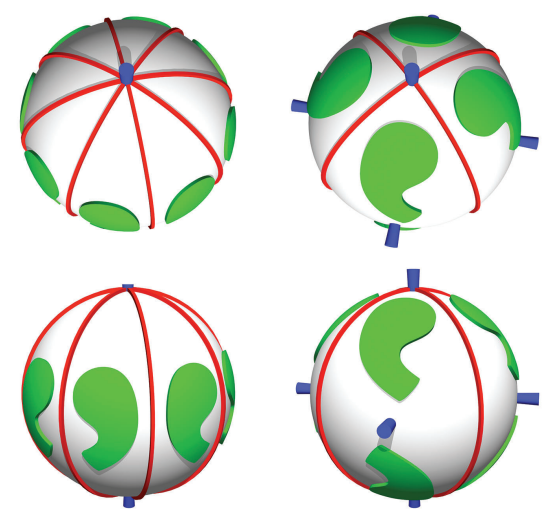

(B) * 44
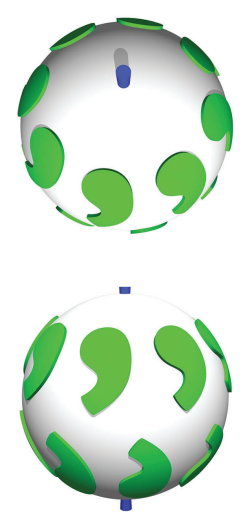

(E) $8 \times$
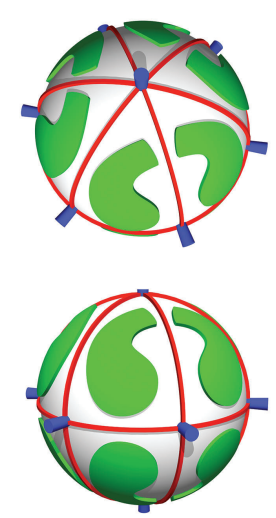

(H) *223

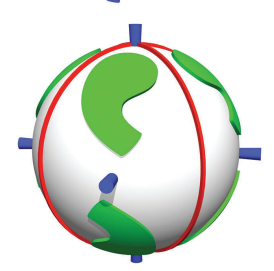

(C) $2 * 2$
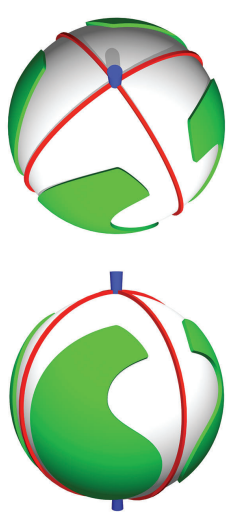

(F) $* 22$
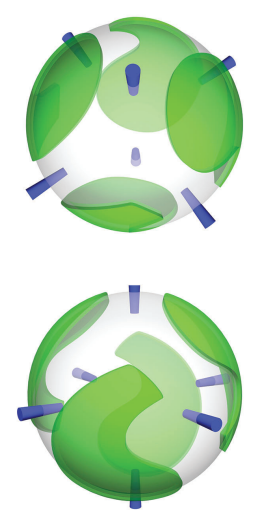

(I) 222
Figure 8. Examples of symmetric objects with their Conway-Thurston orbifold notations. Each object is shown from above and from the side. Points with rotational symmetry are marked in blue, mirror planes in red.

Depending on which is clearer, we render objects in either an opaque or a translucent style.

it acts freely and properly discontinuously on the loop $\sigma$. This implies that the quotient map $\sigma \rightarrow \sigma / H$ is a covering map. The only space that the circle covers is the circle. The group $H$ is then a quotient of the fundamental group of this circle, so is cyclic.
The group $H$ therefore fixes an axis pointwise, and so fixes a pair of antipodal points of the sphere, $a$ and $a^{\prime}$. By the claim, $\sigma$ cannot meet either $a$ or $a^{\prime}$. Furthermore, we claim that $\sigma$ separates $a$ and $a^{\prime}$. To see this, consider the quotient $S^{2} / H$. This is again a two-sphere with two special points, which we denote by $\bar{a}$ and $\bar{a}^{\prime}$. If $\sigma / H$ separates $\bar{a}$ from $\bar{a}^{\prime}$, then we are done. If not, choose a path $\lambda$ from $\bar{a}$ to $\bar{a}^{\prime}$ missing $\sigma / H$. The lifts of $\lambda$ divide the sphere into at least two components, each of which meets $\sigma$. This contradicts the fact that $\sigma$ is connected.

If $G=H$ (symmetry type $n n$ ), then we are done. Otherwise, $G$ is obtained from $H$ by adding a single orientationreversing isometry and taking the group closure. The new element must fix the axis setwise, or else conjugating by the reflection would produce new rotations. It also cannot swap $a$ with $a^{\prime}$, since they lie in non-isometric components of the complement of $\sigma$. Thus $G$ again fixes the axis. (The new symmetry type is * $n n$. Not swapping $a$ with $a^{\prime}$ rules out types $n^{*}$ and $n \times$.)

Finally, suppose that $H$ is infinite. Then it is a closed infinite subgroup of $\mathrm{SO}(3)$, so is therefore either all of $\mathrm{SO}(3)$, or isomorphic to $\mathrm{SO}(2)$. The former is impossible since a piecewise smooth curve does not meet every point of the sphere. Thus $H$ is isomorphic to $\mathrm{SO}(2)$ and $\sigma$ is a (round) circle. Since $\sigma$ does not equally divide the sphere, it is not a great circle. Thus the symmetry group $G$ of $\sigma$ fixes its axis.

These results would seem to imply that it will necessarily be difficult to design an apparatus that will stay on the stage without the performer having to stop and reverse direction. However, if we generalise our construction to allow multiple loops, then we can sidestep the dichotomy of Theorems 4.2 and 4.3 .

\section{Train Tracks}

The idea is to replace the curve $\sigma$ with a graph, $\Sigma$ say, embedded on the rolling sphere. This avoids the problem of Theorem 4.3: the graph $\Sigma$ may contain various loops that split the sphere unevenly, while the whole graph (and therefore the apparatus) has a symmetry type satisfying the hypothesis of Theorem 4.2. The apparatus then has the desired center of mass. The performer chooses which paths on the sphere to roll along, and may change the facing of the apparatus by rolling around an unevenly splitting loop. The construction given in Section 2 works equally well for a graph $\Sigma$ on the rolling sphere.

We add the following further restrictions to our graphs:

1. The paths between vertices of $\Sigma$ are smooth, with tangent directions parallel where two paths meet at a vertex.

2. At each vertex $v$ there must be at least one path approaching $v$ from either of the two sides. 


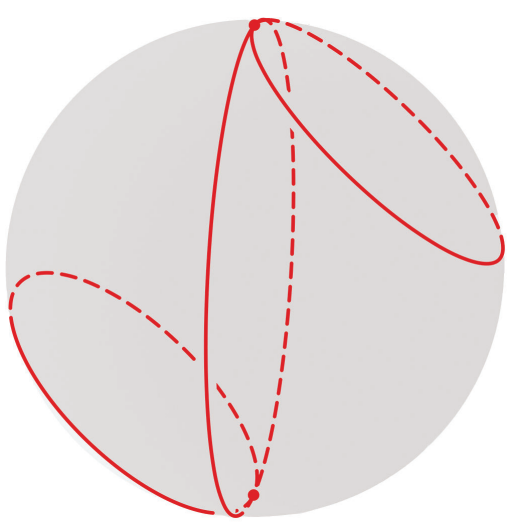

(A) Train tracks for the 270-rolling design.

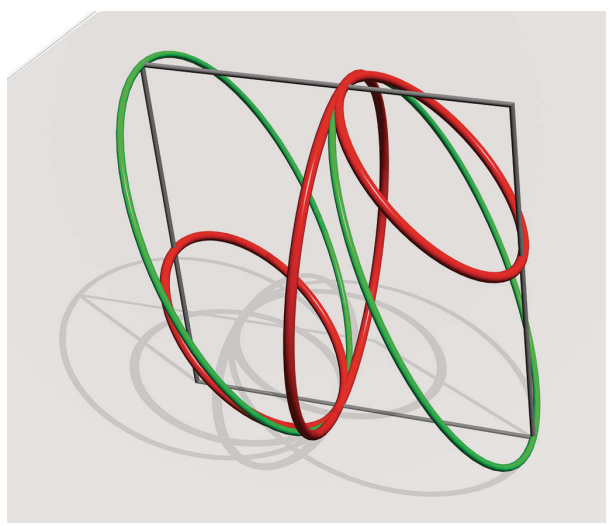

(B) The 270-rolling apparatus (rails in green, rulings in grey).

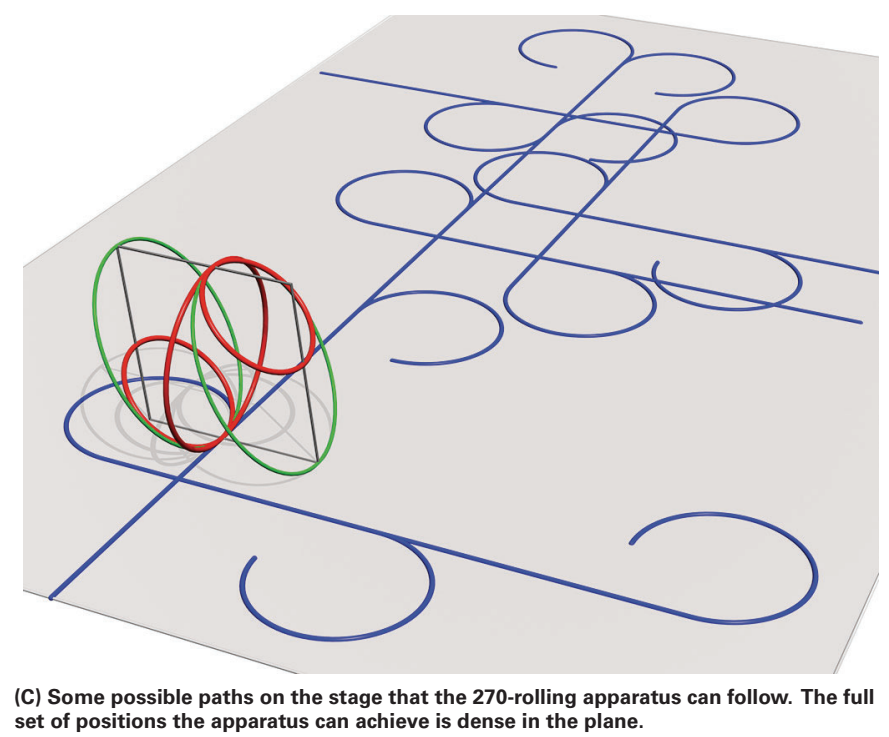

Figure 9. The 270-rolling apparatus.

The first requirement forbids sharp corners in the path. Travelling through any such corner would require the performer slow to a stop, which is almost as bad as Marco's original complaint about the Zigrolling apparatus-that the performer has to stop and go back the other direction. If one travels through a vertex disobeying the second requirement, then one indeed has to stop and go back the other direction. These two restrictions turn the graph into a train track. Train tracks were introduced by Bill Thurston to study surface automorphisms [2].

There are infinitely many possible train track designs that have a symmetry type satisfying the hypothesis of Theorem 4.2. To restrict the possibilities, we are specifically interested in designs that could be physically constructed. Thus, there should be a relatively small number of rails in the design. Moreover, each rail is made from metal pipe bent into the correct shape with a specialized machine, so we prefer simple rail shapes. In the next few sections we give some designs for which $\Sigma$ consists of a small number of arcs of circles. The ruled surfaces corresponding to these arcs are cones and cylinders. The rails are intersections between these cones and cylinders, meaning that they are at worst arcs of ellipses. (Even these were non-trivial to make for the fabricators of the apparatus described in Section 5.3.)

5.1. 270-rolling apparatus. Our first design allows for straight line motion, as in a German wheel, but also allows turns by $270^{\circ}$, hence the name. To that end, the graph $\Sigma$ contains a great circle, together with smaller circles, with radius chosen to achieve the turns by $270^{\circ}$. In particular, by Theorem 3.2 we need the area contained by each small circle to be $\pi / 2$. Solving for this value in equation (5), we get that the radius $r$ of the small circles is $r=\sqrt{7} / 4$ (where as usual, we assume that the rolling sphere has radius one). See Figure 9A. The symmetry of this design is $2^{*}$ (see Figure $8 \mathrm{G})$.

Following the construction in Section 2, we get the design shown in Figure 9B. Again we have included some 


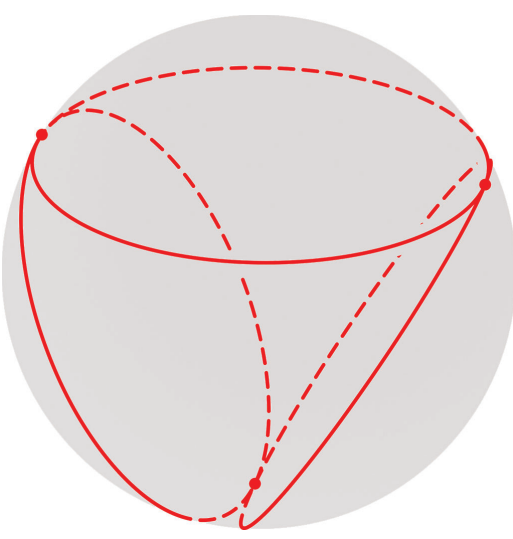

(A) Train tracks for the three rings design.

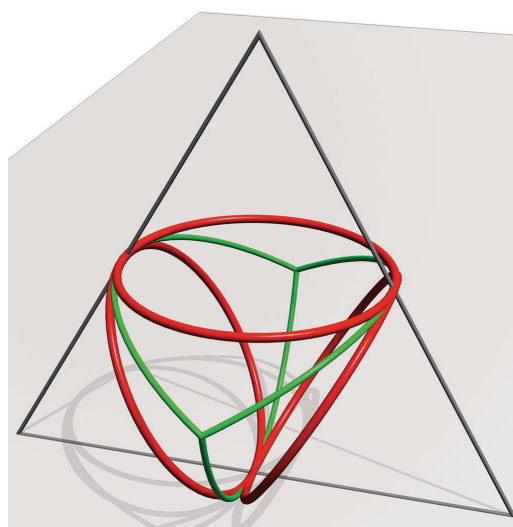

(B) The three rings apparatus (rails in green, rulings in grey).

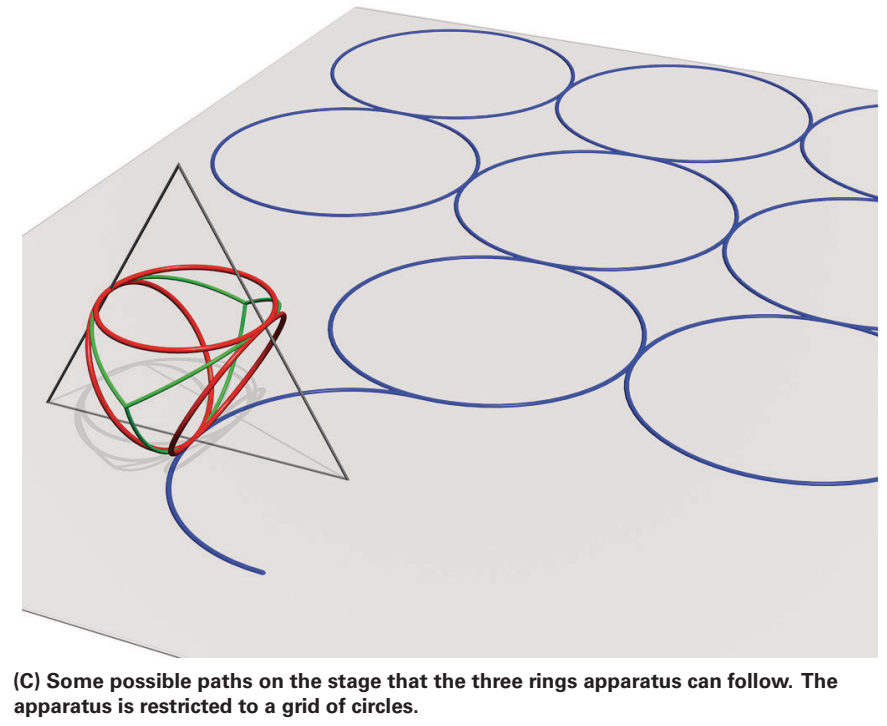

Figure 10. The three rings apparatus.

rulings to connect the rails together. Each rail is the intersection between a cone and a tangent cylinder, which is an ellipse. Also note that in generating the intersection of the ruled surface with itself, we must include the vertices of cones over the small circles. In Figure 9B these are at two of the corners of the parallelogram formed by the included rulings.

Figure 9C shows some possible paths that the apparatus can take. At the illustrated position, assuming that it is rolling away from us, it may either continue rolling in a straight line, or turn to the left by $270^{\circ}$. If it does so, it returns to the same state (in terms of which point of the sphere is touching the stage), but has moved and changed its facing on the stage. Alternatively, it can roll forwards by half a turn, at which point it has the option to turn to the right by $270^{\circ}$. These together allow the apparatus to go more-or-less anywhere on an infinite stage, or keep turning around to stay within a (sufficiently large) bounded area.
Note that the distance that the apparatus rolls forwards after a half turn is $\pi$, while the radius of each of its $270^{\circ}$ turns is $4 / 3 \sqrt{7} / 4=\sqrt{7} / 3$. Since these are not rationally related ( $\pi$ is transcendental, while $\sqrt{7} / 3$ is algebraic), the set of possible locations that the apparatus can get to on an infinite stage is in fact dense.

5.2. Three rings apparatus. Our next design is based on an observation about the circular loop on the rolling sphere that corresponds to a $180^{\circ}$ turn. As before, using Theorem 3.2 this means that the area within the circle is $\pi$. Solving for this value in equation (5), we get that the radius $r$ of the circle is $r=\sqrt{3} / 2$. It happens that three such circles can precisely fit around a great circle of the rolling sphere. See Figure 10A. The three circles are tangent to each other so they form a train track, and the symmetry of the design is *223 (see Figure $8 \mathrm{H}$ ).

We generate rails as in Section 2 and obtain the apparatus shown in Figure 10B. Again, we have added some 

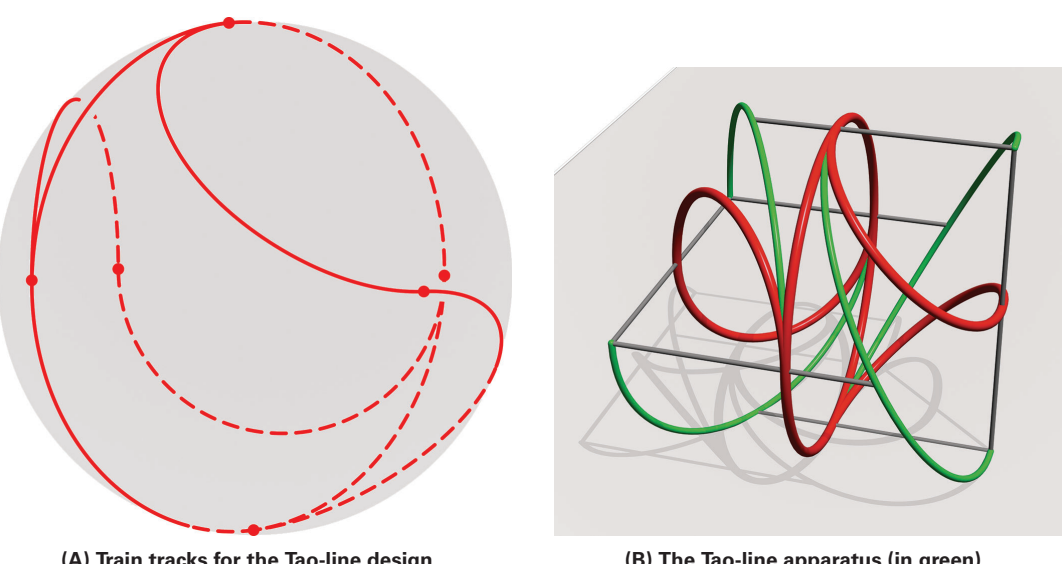

(B) The Tao-line apparatus (in green).

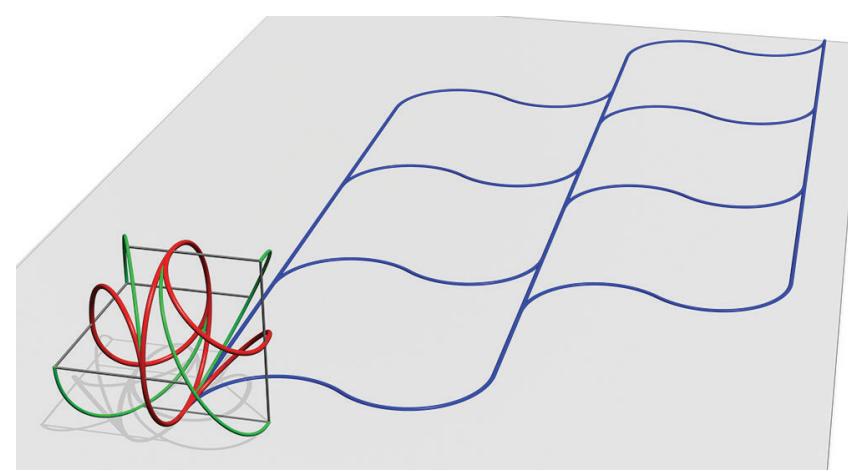

(C) Some possible paths on the stage that the Tao-line apparatus can follow.

Figure 11. The Tao-line apparatus.

rulings; the endpoints of these rulings are at the vertices of cones over the three circles. As before, for a full-size apparatus these would not be suitable. Here in particular, the rulings make the structure much larger than with the rails alone. The vertex of the cone over each circle could be replaced with a rail in the form of a circular cross-section through the cone, parallel to the circle of $\Sigma$.

Rolling halfway around one of the circles, from one vertex of the train track to another, has the effect of rolling around a quarter circle on the stage. By alternating right and left turns, this apparatus approximates the movement of a Zigrolling apparatus, although of course the performer may choose some other path at any vertex of the train track. It is remarkable that this design has very different and yet simultaneously discrete and regular paths on the sphere and the plane.

5.3. Tao-line apparatus. Our final design does not solve Marco's problem of the performer having to stop and go backwards, but it has other interesting properties. The train track $\Sigma$ shown in Figure 11A has a great circle, which again means that the corresponding apparatus can be used as a German wheel. Two additional "S"-shaped paths are each made from two semicircles, drawing a Yin and Yang symbol on each hemisphere. These S-paths remove any reflection symmetries and reduce the symmetry type to 222 (see Figure 8I). Following an S-path returns one to the great circle, but travelling in the opposite direction. The performer can roll forwards along the great circle, follow the S-path, and end up rolling backwards along the great circle. This happens without stopping the apparatus to change direction, or the performer turning around within the apparatus.

Theorem 3.2 implies that the turning angle going around one of the Yin or Yang loops is $\pi$. Note however that this calculation assumes that the loop starts and ends going in the same direction. Doing this would require that we make an illegal "hairpin" turn by $\pi$. If instead we go around the loop and end up going in the opposite direction around the great circle, then our turning angle is zero, as shown in Figure 11C. Thus we do not solve Marco's problem! Instead, the apparatus becomes a German wheel with the ability to "change lanes" by following an S-path.

This design caught the eye of Lee Brasuell, a professor in the Department of Theatre at Oklahoma State University, and an expert in circus apparatus. I met Lee at a new-faculty orientation event in 2014; he later named this apparatus the "Tao-line" (referencing the Yin and Yang symbol's roots in Taoism). He was also attracted to the 


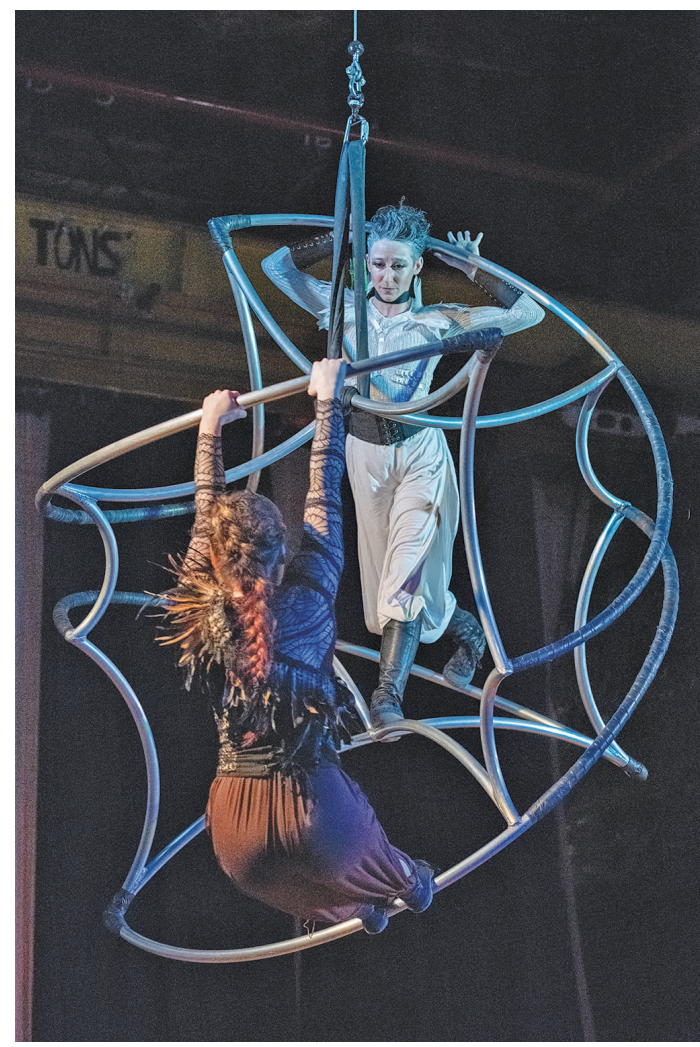

(A) Performers Shayna Golub and Sara Zepezauer.

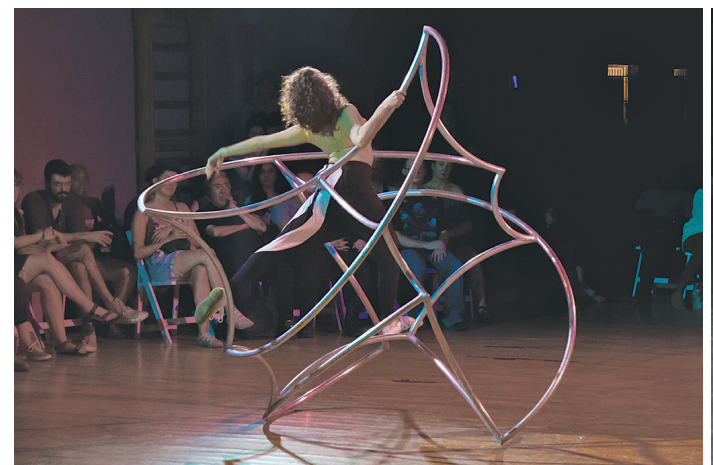

(C) Performer Ayla Weisz.

Figure 12. The Tao-line apparatus.

varied visual appearances of the apparatus as seen from different directions, and saw its potential as both a groundbased and an aerial apparatus (that is, hung from the ceiling). One thing led to another, and Lee developed the Tao-line design into a full-size physical apparatus; see Figure 12. The rails of the design consist of four half-ellipses, which in Figure 11 are connected together by four line segments from the ruled surface. For the full-scale apparatus, we again replace these connecting straight lines with various arcs that curve in towards the center of the apparatus.

The apparatus was funded in part by an Oklahoma DaVinci Fellowship and the Mary Lou Lemon Endowment

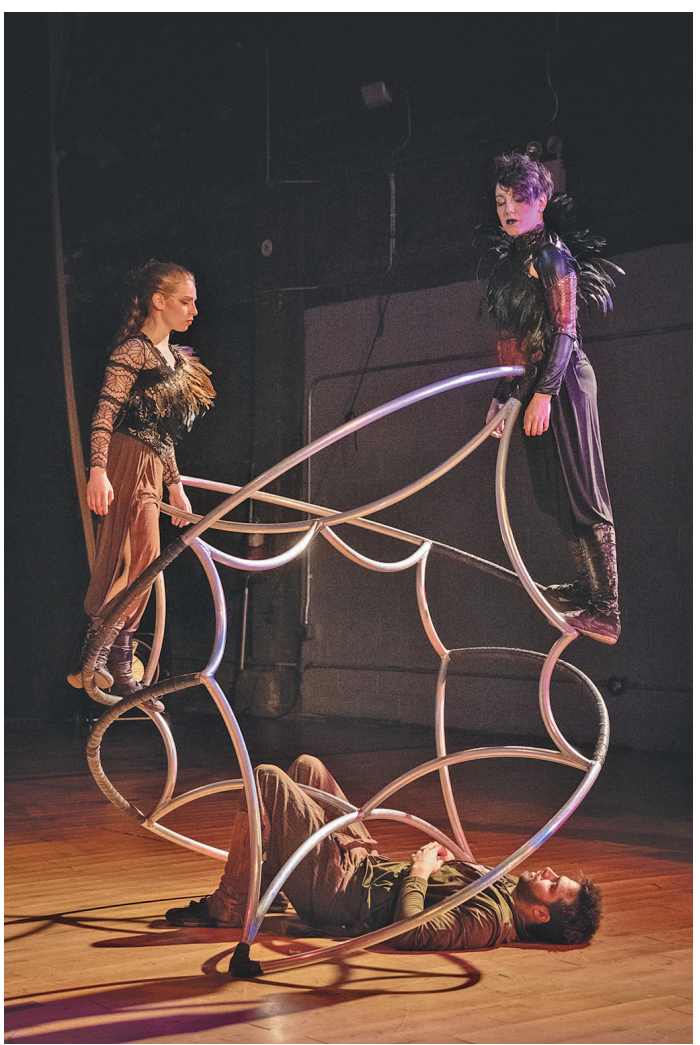

(B) Performers Shayna Golub, Angela Butch, and Chris Delgado.

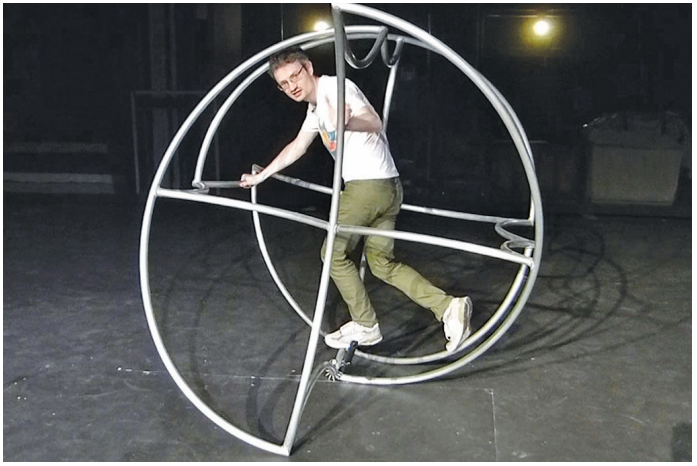

(D) The author.

for Underrepresented Voices. It was fabricated by Chicago Flyhouse, a speciality rigging company based in Chicago, Illinois.

Before the pandemic, the apparatus was workshopped and performed with at The Muse Brooklyn, a community and performance space in Brooklyn, New York. Brief videos can be viewed at https://bit.7y/2RJXsuU and https://bit. 1y/3gnCk63. Performers have discovered many new ways to play with the apparatus, both on the ground and in the air. The specific symmetry of the apparatus was not particularly important to the mathematical design process, but seems to be very appealing in 
performance: the many points of 2-fold rotational symmetry each allow it to naturally accommodate two acrobats in a "seesaw" configuration, as seen in Figures 12A and 12B. Another unanticipated aspect of the apparatus is the difficulty of gracefully rolling it where two tracks diverge away from a vertex of the train track: it does not take much to jump the apparatus from one track to the other, producing an audible "clunk." Performers have learned how to position themselves near a vertex of the graph to cleanly stay on their desired track. With the end of the pandemic now apparently in sight, we hope that acrobats will be able to continue exploring and performing with the Tao-line apparatus.

ACKNOWLEDGMENTS. The author thanks Marco Paoletti for telling him about the problem that inspired this paper, Rémi Coulon, Sabetta Matsumoto, Saul Schleimer, Steve Trettel, and Toni Vighetto for helpful conversations, Lee Brasuell for realising one of this project's designs at full scale, and the referees for their very useful comments. The author was supported in part by National Science Foundation grants DMS1308767 and DMS-1708239.

\section{References}

[1] John H. Conway, Heidi Burgiel, and Chaim GoodmanStrauss, The symmetries of things, A K Peters, Ltd., Wellesley, MA, 2008. MR2410150

[2] R. C. Penner and J. L. Harer, Combinatorics of train tracks, Annals of Mathematics Studies, vol. 125, Princeton University Press, Princeton, NJ, 1992, DOI 10.1515/9781400882458. MR1144770

[3] Ian Stewart, Cone with a twist, Scientific American 281 (1999), no. 4, 116-117.

[4] Dirk J. Struik, Lectures on classical differential geometry, Addison-Wesley Press, Inc., Cambridge, Mass., 1950. MR0036551

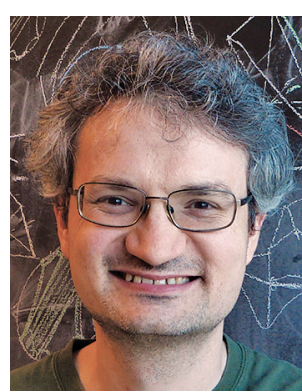

Henry Segerman

Credits

Opening graphic is courtesy of Marco Paoletti.

Figure 1 is courtesy of Tony Vighetto.

Figures 2-11 are courtesy of Henry Segerman.

Figures $12 \mathrm{~A}$ and $12 \mathrm{~B}$ are courtesy of Tony Falcone.

Figure $12 \mathrm{C}$ is courtesy of Kayla.

Figure 12D is courtesy of Lee Brasuell.

Photo of Henry Segerman is courtesy of Sabetta Matsumoto.

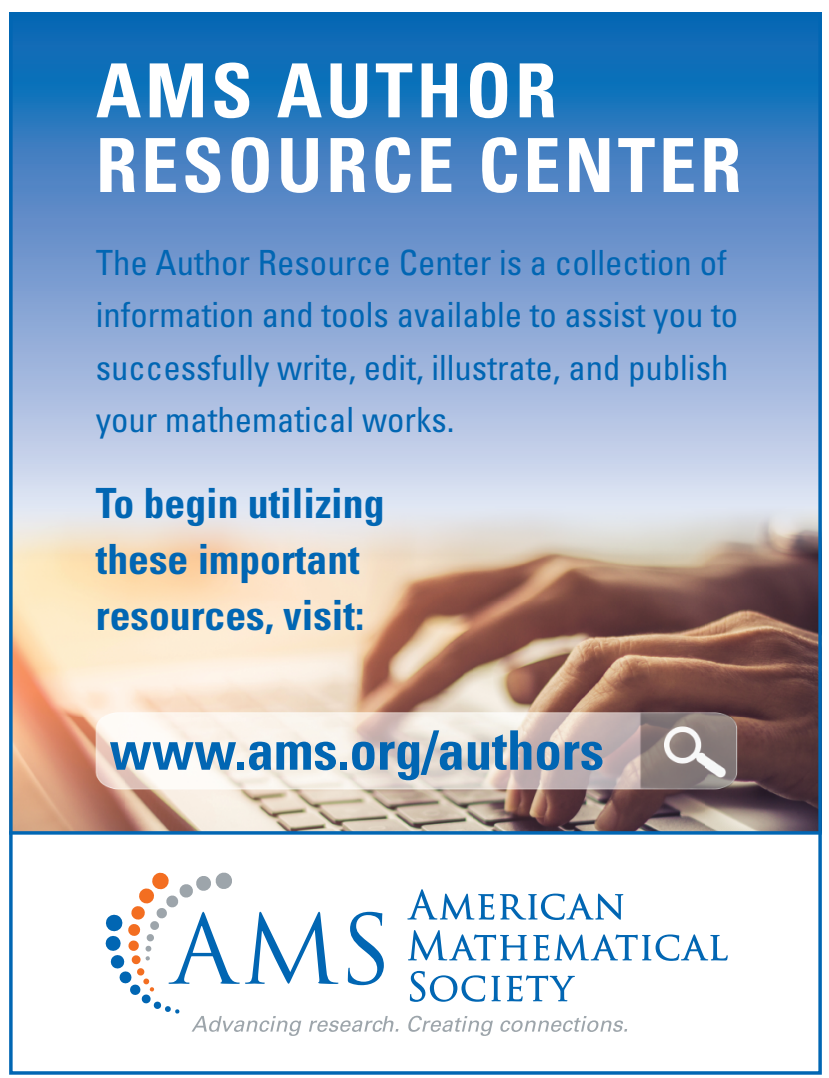

